\title{
Detecting Local Events Using Global Sensing
}

\author{
Mahsan Rofouei, Majid Sarrafzadeh, Miodrag Potkonjak \\ Computer Science Department \\ University of California, Los Angeles \\ Los Angeles, USA \\ \{mahsan, majid, miodrag\}@cs.ucla.edu
}

\begin{abstract}
In order to create low power, low latency and reliable sensing systems, we propose a sensing strategy that identifies local events by the means of global measurements. We claim that capturing events globally, although seems against intuition, can save energy by enabling the organization of effective searching queries. To enable this capability, sensor readings can be combined using electronic switches and therefore enable event detections in groups of sensors with single measurements. We demonstrate this sensing mechanism on a prototype keyboard system made from E-Textile pressure sensors.
\end{abstract}

\section{INTRODUCTION}

Maximizing the lifetime of sensing systems by minimizing their energy consumption is considered important in design of sensing systems. Some of these systems are required to collect samples at all times, and events may occur at any moment of time without a known pattern. Therefore, it is crucial to design intelligent sampling techniques that are capable of capturing all events with minimal energy required.

In this paper we show that global sensing can help to minimize the number of samples needed to localize the position of events. Our power consumption model suggests that coarse grained measurements that use fewer measurements to find events are beneficial in minimizing the amount of energy. By using electronic switches, sensing locations can be combined to enable group sensing. We also use methods such as interleaved sampling and processing that enables organizing intelligent sampling schedules based on observation from sampling a subset of sensors through global sensing.

Smart E-Textiles provide a good platform for design and implementation of pressure sensor arrays for use in a wide spectrum of applications. Due to their flexible and fabric feel nature they can be used in smart clothing items, medical shoe insoles, bed sheets and many more areas [5, 6, 21]. We study the use of E-Textiles in the design of a soft portable keyboard and design global sensing techniques to detect key presses on the keyboard. This keyboard can be used with wireless devices such as smart phones and Personal Digital Assistants (PDAs). This enables light, low cost and personalized keyboards that can be used with multiple devices with adequate keys to perform fast and easy interactions with small wireless systems.

The rest of the paper is organized as follows: In Section II we present an overview of related work in this area. Section III provides a description of the platform used for our study in addition to the power consumption model used. Global sensing techniques such as binary sensors and interleaved sampling and processing are described in Section IV. We show the effectiveness of methods presented in design of a soft E-Textile keyboard in Section $\mathrm{V}$ and finally draw conclusions in Section VI.

\section{RELATED WORK}

Electronic textiles or smart fabrics [3] have enabled a good platform for pervasive computing. The work in [2] provides an overview of efforts and challenges associated with building systems that have sensors and computational elements embedded into E-Textiles with the goal of gathering sensitive information and monitoring. SmartShirt [4] is an example of such systems that measures human heart rhythm and respiration with conductive fiber grid and sensors fully integrated in the shirt. E-Textiles are used in sheets and pillows in order to monitor sleep in [5]. A smart E-Textile based soft keyboard is presented in [6].

In the last two decades energy emerged as one of the most important design metrics [22-25]. Energy efficiency and optimizing sampling techniques in sensor networks and embedded sensing systems has received a great deal of attention [7, 8]. Design of optimal periodic sampling techniques for embedded sensing environments has been studied in $[11,12]$. Sampling problems in event-driven sensor sampling techniques have been addressed in $[9,10]$. Recently, several distributed event-triggered sampling strategies have been proposed [15].

We use the global sensing method presented in the design of an E-Textile keyboard. There has been a great amount of research performed in developing optimized keyboards by techniques such as studying typing characteristics $[16,17,18]$. In addition, different technologies have been used to develop soft keyboards [19, 20]. 


\section{PRELIMINARIES}

In this section we describe characteristics of E-Textiles and how they can be used in building pressure sensor arrays. Next, we describe the power consumption model used in our studies. This model shows why global sensing is beneficial.

\section{A. E-Textile Technology}

E-Textiles [3] are composite yarns made of fibers coated with piezo-electric polymer. They offer a good platform for design of high density and low cost pressure sensor arrays. ETextile pressure sensors can be built using a three stacked layer structure where E-Textile material is sandwiched between two layers of conductive fabric. In the event of applied force on the surface of this pressure sensor, the intra fibers within the E-Textile material are squeezed together, resulting in a smaller throughout resistor. Figure 1 shows the relation between resistance and force in an E-Textile pressure sensor.

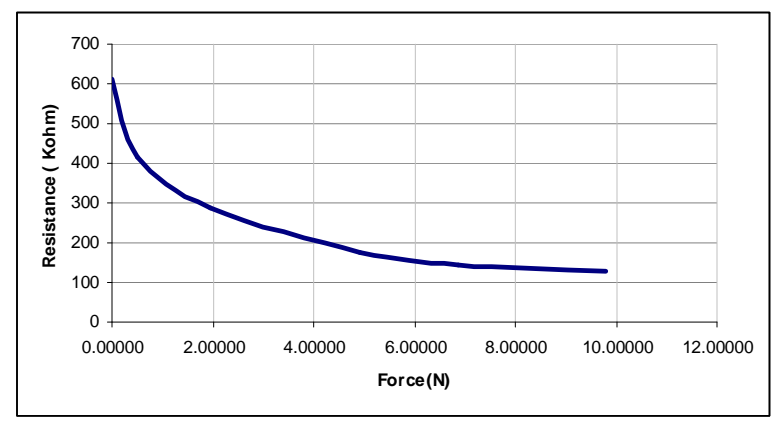

Figure 1. Resistance response based on applied force

\section{B. Power Consumption Model}

Power consumption in sensing systems is mainly due to three tasks: 1) Data transmission to host, 2) Power consumed by the sensing circuit and 3) Sampling power. Table $1[13,14]$ shows the energy consumption of these three tasks in our platform. Our platform uses E-Textiles in the sensing circuit and MSP430f2274 microcontroller with CC2500 wireless chip.

TABLE I. ENERGY CONSUMPTION OF SUB-MOdULES.

\begin{tabular}{|c|c|}
\hline Sub-module & Energy (J) \\
\hline ADC ( per sample) & $9 \times \mathrm{E}-9$ \\
\hline Data Transmission( per sample) & $7.2 \times \mathrm{E}-9$ \\
\hline Sensing circuit (average per sensor) & $4.8 \times \mathrm{E}-10$ \\
\hline
\end{tabular}

Table 1 shows that ADC energy consumption dominates the sensing circuit energy consumption. Therefore, in order to minimize total energy consumed for sensing, it is beneficial to use as less ADC units as possible. This motivates the idea of global sensing which combines sensor locations and performs only a single reading instead of multiple readings. Table 1 also suggests that localized processing is a must since data transmission energy is in the order of ADC energy.

\section{GLOBAL SENSING}

The results of Section II.B suggest that based on power levels during sensing, combing sensing locations into a single sensor and performing group readings is beneficial as it requires only a single $\mathrm{ADC}$ unit. In this section, we propose techniques for global sensing that reduces the number of samples required. In this work, we are interested in finding the location of a single event in a sensor array using minimum number of readings.

\section{A. Binary Sensors}

We propose a multi-layer design in which each layer consists of multiple sensing locations. Each layer is a single pressure sensor with the design described in Section II.A. Pressure asserted at the top layer is sensed in the layers below due to the nature of E-Textiles. Relatively lower pressure levels are sensed at lower levels. A calibration stage can be performed at the beginning to obtain pressure levels at each of the layers. This multi-layer design enables sampling schemes that can reduce the number of readings to perceive the location of an event.

Here, we describe techniques that find the location of events, given that the occurrence of an event is known. This occurrence of an event is detected by adding a layer (event detector layer) over all sensing locations with a single pressure sensor that would perceive any event. We use a binary mapping from values 0-255 using a found threshold. Values above this threshold are mapped to 1 and the remaining values are considered 0 . To elaborate, a reading of ' 1 ' in the event detector layer, determines the occurrence of an event

In our proposed multi-layer sensing system we use binary representation of sensors to reduce the number of samples required to deduce the location of an event. The number of layers in the multi-layer sensor array is determined by the number of sensors needed. For an $n$ sensor sensing array, [logn] layers is required. Each layer is responsible for one of the digits of the binary representation of sensors. In each layer, sensor locations that have a ' 1 ' in the digit corresponding to that layer, are connected to each other. To elaborate, in each layer there is a single pressure sensor, connecting the locations of $n / 2$ sensing locations.

Based on the above discussion, [logn] layers can support up to $n$ sensor locations. Figure 2 shows how this approach works for 8 sensor locations. Based on the method described above, the sensor array architecture would be composed of three layers. In layer 1, a single pressure sensor is placed at the positions corresponding to sensors 1 (001), 3 (011), 5 (101) and 7 (111). With same reasoning two other pressure sensors are placed at layers 2 and 3. Therefore, by sampling only the signals coming out of each layer, we are able to conclude the location of an event with only three readings. This saving becomes significant when there is larger number of sensors. 


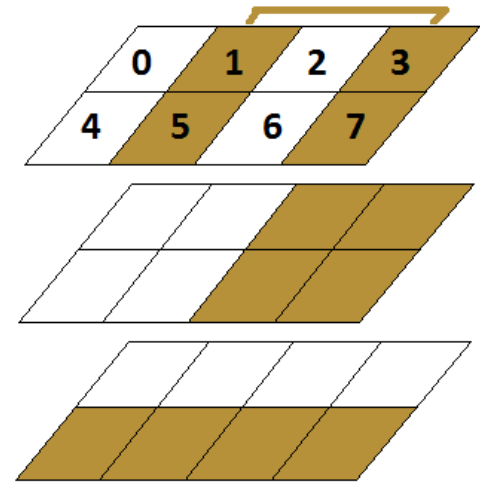

Figure 2. Three layer binary sensors

\section{B. Interleaved Sampling and Processing}

The multi-layer approach can be extended to be used for different purposes to help reduce the number of samples. The technique described in Section IV.A, assumes equal probabilities for all sensor locations. However depending on the application, different locations can have different probabilities of being activated. Additional layers can be included to accommodate for more frequent locations. As an example, if $90 \%$ of the time only one of the locations is activated in the example of Figure 3, it is not beneficial to always perform three readings in the case of an event. It is best to sample only the most frequent location by using an additional layer and only perform the three extra readings when necessary. Using this method the expected number of readings is reduced to 1.3 instead of 3 .

$$
\mathrm{E}_{\text {Readings }}=90 \% \times 1+10 \% \times(3+1)=1.3
$$

Interleaved sampling and processing technique suggests that sampling is performed at different stages. This way sampling schedules can be designed based on the result of the processing performed on the samples collected in the previous stage. In the above example, based on the result of sampling the most frequent location, in the next stage either 0 or 3 samples are performed.

\section{EXPERIMENTAL RESULTS}

We show the effectiveness of the methods proposed in section IV, in the design of an E-Textile soft keyboard with 102 keys. A trivial sampling mechanism would need 101 readings to deduce the key pressed in the case of an event. More intelligent sampling mechanisms such as one where sensors are arranged in rows and columns and by applying voltage at columns of interest all rows are scanned, require 21 readings for the same number of keys. [6] proposes methods that would minimize this number when more time is available for processing.

Using Binary sensors, 102 keys can be arranged into 7 layers $([\log (102)])$. This means that performing 7 readings would identify any key presses. This is large savings even compared to intelligent methods that require 21 readings. Therefore the proposed keyboard consists of the total of 8 layers: 1 for event detection and 8 for detecting the key that caused the event.

Next, we utilize interleaved sampling and processing technique described in section IV.B to further minimize the number of readings. We used relative frequency of letters in English from [1] to understand different probabilities of each key being pressed. Based on [1], keys: 'space', 'e', 't' and 'a' are the four most frequent keys utilized. In this approach, we add an extra layer with a single sensor at the location of the most frequent keys. This layer would be activated if any of the frequent keys are activated. Extra layers are inserted based on the number of frequent keys chosen as most frequent keys.

Table II shows the number of readings required, based on locations included in the additional layer. In each row, keys are added based on highest probability from [1]. The interleaved sampling and processing method, first samples this extra layer using a single reading. If this reading results in a ' 0 ', in the next sampling 7 more readings are performed, resulting in a total of 8 readings for less frequent keys. However if the reading is ' 1 ', depending on what locations are chosen to be placed at the extra layer, different readings are needed to be performed. If it is a single location ( eg. space), then no additional reading is requires. For higher numbers, based on the same binary sensor technique, [logn] readings will be required. Please note that the calculation in Table 1 is static and only performed once and the best configuration determines the design of the sensor array for the soft keyboard.

TABLE II. Most Frequent Key COMBINATIONS

\begin{tabular}{|c|c|c|}
\hline Keys & $\begin{array}{c}\text { Sum of } \\
\text { Probabilities }(\%)\end{array}$ & $\begin{array}{c}\text { Number of } \\
\text { Readings }\end{array}$ \\
\hline None & 0 & 7 \\
\hline Space & 16.45 & 6.84 \\
\hline Space, $e$ & 27.06 & 6.37 \\
\hline Space,e,t & 34.62 & 6.26 \\
\hline Space,e,t,a & 41.44 & 5.92 \\
\hline Space,e,t,a,o & 47.71 & 6.09 \\
\hline Space,e,t,a,o,i & 53.52 & 5.85 \\
\hline Space,e,t,a,o,i,n & 59.15 & 5.63 \\
\hline 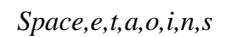 & 64.43 & 6.06 \\
\hline 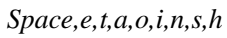 & 69.52 & 5.91 \\
\hline 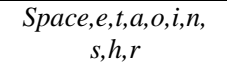 & 74.52 & 5.76 \\
\hline 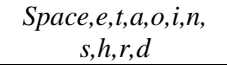 & 78.07 & 5.65 \\
\hline 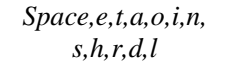 & 81.43 & 5.55 \\
\hline 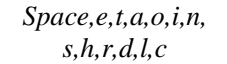 & 83.57 & 5.48 \\
\hline 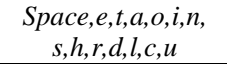 & 86.05 & 5.41 \\
\hline
\end{tabular}




\begin{tabular}{|c|c|c|}
\hline Keys & $\begin{array}{c}\text { Sum of } \\
\text { Probabilities }(\%)\end{array}$ & $\begin{array}{l}\text { Number of } \\
\text { Readings }\end{array}$ \\
\hline 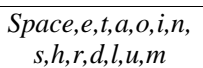 & 88.06 & 5.35 \\
\hline $\begin{array}{l}\text { Space,e,t,a,o,i,n, } \\
s, h, r, d, l, c, u, m, w\end{array}$ & 90.03 & 5.25 \\
\hline 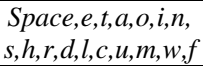 & 91.89 & 6.16 \\
\hline
\end{tabular}

Table II shows that the configuration resulting in minimum number of readings is one that is composed of keys: " Space, $\mathrm{e}, \mathrm{t}, \mathrm{a}, \mathrm{o}, \mathrm{i}, \mathrm{n}, \mathrm{s}, \mathrm{h}, \mathrm{r}, \mathrm{d}, \mathrm{l}, \mathrm{c}, \mathrm{u}, \mathrm{m}, \mathrm{w}$ " and requires expected number of 5.25 readings. This method can be adapted to other languages as well and customized keyboards can be made for different languages.

\section{CONCLUSION}

We have proposed a sensing strategy that uses global sensing in order to localize events in sensing systems. This method can be applied to many embedded sensing systems. We chose E-Textiles as a platform to develop sensor arrays since due to its nature enables layered design. Techniques such as using binary sensors and interleaved sampling and processing result in large savings in the amount of reading performed. We demonstrate the efficiency of these techniques in the design of an E-Textile soft keyboard.

\section{REFERENCES}

[1] H. Beker, and F. Piper, "Cipher Systems: The Protection of Communications", Northwood Books, London. 1982.

[2] Marculescu D et al. "Electronic Textiles: A Platform for Pervasive Computing", Proceedings of the IEEE 2003; 91 (12).

[3] http://www.eeonyx.com/

[4] D. Marculescu, R. Marculescu, N.H. Zamora, P. Stanley-Marbell, P.K. Khosla, S. Park, S. Jayaraman, S. Jung, C. Lauterbach, W. Weber, T. Kirstein, D. Cottet, J. Grzyb, G. Troster, M. Jones, T. Martin, and Z. Nakad, "Electronic textiles: A platform for pervasive computing," Proc. IEEE, vol. 91, no. 12, pp. 1991-2016, Dec. 2003.

[5] S. Devot, A. M. Bianchi, et al, "Sleep Monitoring Through a Textile Recording System", Proceedings of the 29th Annual International Conference of the IEEE EMBS, Lyon, France, Aug 2007.

[6] M. Rofouei, M. Potkonjak, M. Sarrafzadeh, "Energy Efficient E-Textile Based Portable Keyboard", International Symposium on Low Power Electronics and Design (ISLPED) Aug 2011.

[7] D. Ganesan, B. Krishnamachari, et al. , "Complex Behavior at Scale: An Experimental Study of Low-Power Wireless Sensor Networks", Technical Report UCLA/CSD-TR 02-0013, 2002.

[8] Joseph Polastre, Jason Hill, et al. ,"Versatile low power media access for wireless sensor networks", Proceedings of the 2nd international conference on Embedded networked sensor systems, 2004.
[9] M. Mailinowski, M. Moskwa, et al, "CargoNet: a low-cost micropower sensor node exploiting quasi-passive wakeup for adaptive asynchronous monitoring of exceptional events", Proceedings of the 5th international conference on embedded networked sensor systems, 2007.

[10] Sasha Jevtic , Mathew Kotowsky, et al. , "Lucid dreaming: reliable analog event detection for energy-constrained applications", Proceedings of IPSN, 2007.

[11] M. A. Batalin, M. Rahimi, et al., "Call and response: experiments in sampling the environment", Proceedings of the 2nd international conference on Embedded networked sensor systems, 2004.

[12] S. Gandhi , S. Suri , E. Welzl, "Catching elephants with mice: sparse sampling for monitoring sensor networks", Proceedings of the 5th international conference on Embedded networked sensor systems, 2007.

[13] http://focus.ti.com/docs/prod/folders/print/msp430-adc10.html

[14] [18] http://focus.ti.com/docs/toolsw/folders/print/ez430-rf2500t.html

[15] P. Wan and M.D. Lemmon. Event-triggered distributed optimization in sensor networks. In Information Processing in Sensor Networks (IPSN), 2009.

[16] I.S. MacKenzie, "Fitts' law as a research and design tool in humancomputer interaction, Human- Computer Interaction", Vol. 7, pp. 91139,1992

[17] W. Soukoreff, I.S. MacKenzie, "Theoretical upper and lower bounds on typing speeds using a stylus and keyboard", Behaviour \& Information Technology Vol. 14, pp. 370-379, 1995.

[18] S. Zhai, A. Sue, J. Accot, "Movement model, hits distribution and learning in virtual keyboarding", SIGCHI conference on Human factors in computing systems, pp. 17-24, 2002.

[19] I.S. MacKenzie, S.X. Zhang, "The design and evaluation of a highperformance soft keyboard", SIGCHI conference on Human factors in computing systems, pp. 25-31, 1999.

[20] M. Hunter, S. Zhai, B.A. Smith, "Physics-based graphical keyboard design", CHI Human factors in computing systems, pp. 157-158, 2000.

[21] M. Rofouei , W. Xu and M. Sarrafzadeh "Computing with uncertainty in a smart textile surface for object recognition", Proc. Int. Conf. Multisensor Fusion Integr. Intell. Syst., pp.256 - 261 , 2010.

[22] F. Koushanfar, A. Davare, D. Nguyen, A. Sangiovanni-Vincentelli, M. Potkonjak, "Techniques for Maintaining Connectivity in Wireless Adhoc Networks Under Energy Constraints", ACM Transaction on Embedded Computing Systems", vol. 6, no. 3, pp. 16-26, 2007.

[23] A.P. Chandrakasan, M. Potkonjak, J. Rabaey, R. Brodersen, "HyperLP: A Design System for Power Minimization using Architectural Transformations", ICCAD-92 IEEE International Conference on Computer-Aided Design, Santa Clara, CA, pp. 300-303, November 1992.

[24] I. Hong, M. B. Srivastava, M. Potkonjak, "On-Line Scheduling of Hard Real-Time Tasks on Variable Voltage Processor", ICCAD98 International Conference on Computer-Aided Design, pp. 653-656, San Jose, CA, November 1998.

[25] I. Hong, G. Qu, M. Potkonjak, M.B. Srivastava, "Synthesis Techniques for Low-Power Hard Real-Time Systems on Variable Voltage Processor", 1998 Real-Time System Symposium, pp. 178-187, Madrid, Spain, December 1998. 